Journal of

Molecular Microbiology

and Biotechnology
J Mol Microbiol Biotechnol 2007;12:20-32

DOI: $\underline{10.1159 / 000096456}$

\title{
The Phosphotransferase System of Lactobacillus casei: Regulation of Carbon Metabolism and Connection to Cold Shock Response
}

\author{
Vicente Monedero ${ }^{a} \quad$ Alain Mazé ${ }^{b, c} \quad G^{2}$ gory Boël ${ }^{b, c} \quad$ Manuel Zúñiga $^{a}$ \\ Sophie Beaufils ${ }^{\text {b, c }}$ Axel Hartke ${ }^{b}$ Josef Deutscher ${ }^{c}$ \\ a Departamento de Biotecnología, Instituto de Agroquímica y Tecnología de Alimentos, CSIC, Burjassot, Spain; \\ ${ }^{b}$ Laboratoire de Microbiologie de l'Environnement (EA956 USC INRA 2017), IBFA, Université de Caen, Caen, and \\ 'Microbiologie et Génétique Moléculaire, CNRS/INRA/INA-PG UMR2585, Thiverval-Grignon, France
}

\section{Key Words}

Lactobacillus casei P Phosphoenolpyruvate:carbohydrate phosphotransferase system • Carbon catabolite repression • Inducer exclusion $\cdot$ Inducer expulsion

\section{Abstract}

Genome sequencing of two different Lactobacillus casei strains (ATCC334 and BL23) is presently going on and preliminary data revealed that this lactic acid bacterium possesses numerous carbohydrate transport systems probably reflecting its capacity to proliferate under varying environmental conditions. Many carbohydrate transporters belong to the phosphoenolpyruvate:sugar phosphotransferase system (PTS), but all different kinds of non-PTS transporters are present as well and their substrates are known in a few cases. In $L$. casei regulation of carbohydrate transport and carbon metabolism is mainly achieved by PTS proteins. Carbon catabolite repression (CCR) is mediated via several mechanisms, including the major P-Ser-HPr/catabolite control protein A ( $\mathrm{CcpA}$ )-dependent mechanism. Catabolite response elements, the target sites for the P-Ser-HPr/CcpA complex, precede numerous genes and operons. PTS regulation domain-containing antiterminators and transcription activa- tors are also present in both L. casei strains. Their activity is usually controlled by two PTS-mediated phosphorylation reactions exerting antagonistic effects on the transcription regulators: P $\sim$ ElIB-dependent phosphorylation regulates induction of the corresponding genes and $\mathrm{P} \sim \mathrm{His}-\mathrm{HPr}$-mediated phosphorylation plays a role in CCR. Carbohydrate transport of $L$. casei is also regulated via inducer exclusion and inducer expulsion. The presence of glucose, fructose, etc. leads to inhibition of the transport or metabolism of less favorable carbon sources (inducer exclusion) or to the export of accumulated non-metabolizable carbon sources (inducer expulsion). While P-Ser-HPr is essential for inducer exclusion of maltose, it is not necessary for the expulsion of accumulated thio-methyl- $\beta-D$-galactopyranoside. Surprisingly, recent evidence suggests that the PTS of $L$. casei also plays a role in cold shock response.

Copyright $\odot 2007$ S. Karger AG, Basel

\section{Introduction}

Lactobacillus case $i$ is a Gram-positive facultatively anaerobic and heterofermentative lactic acid bacterium. In the past, the classification of this species was often con-

\section{KARGER}

Fax +41613061234

E-Mail karger@karger.ch

www.karger.com (c) 2007 S. Karger AG, Basel

$1464-1801 / 07 / 0122-0020 \$ 23.50 / 0$

Accessible online at:

www.karger.com $/ \mathrm{mmb}$
Josef Deutscher

Microbiologie et Génétique Moléculaire, CNRS/INRA/INA-PG

FR-78850 Thiverval-Grignon (France)

Tel. +33 13081 5447, Fax +33130815457

E-Mail Josef.Deutscher@grignon.inra.fr 
fusing and strains of L. casei, Lactobacillus rhamnosus, Lactobacillus paracasei and Lactobacillus zeae were frequently mixed up and had to be reclassified. Most importantly, the previous L. casei-type strain ATCC393 has been identified as L. zeae [Dicks et al., 1996] and a new $L$. casei-type strain (ATCC334) has been proposed [Dellaglio et al., 2002]. The genomes of two L. casei strains (ATCC334 and BL23, the latter has been widely used for genetic, biochemical and physiological studies) have been partly sequenced [Klaenhammer et al., 2002]. Genes present in both organisms are nearly identical (more than 98\% identity), but the genome of BL23 is significantly larger and contains numerous insertions, which probably account for most physiological differences between the two strains. The preliminary sequencing data allow a better understanding of the regulatory mechanisms controlling carbohydrate transport, carbon metabolism and stress response in L. casei species. The understanding of these mechanisms is of great biotechnological importance, since $L$. casei strains are widely used in numerous fermentation processes. In addition, some $L$. casei strains exhibit probiotic effects and several $L$. casei-containing health-promoting products (L. casei Shirota, L. casei defensis, etc.) [Coeuret et al., 2004] have been commercialized. In the following review we try to summarize the knowledge about regulatory mechanisms controlling carbohydrate transport, carbon metabolism and cold shock response in L. casei. Among others they include carbon catabolite repression (CCR), inducer exclusion and inducer expulsion.

Most of these phenomena are controlled by the phosphoenolpyruvate (PEP):carbohydrate phosphotransferase system (PTS) via PEP- or ATP-dependent phosphorylation of its protein components. The PTS is usually composed of the two general proteins EI and HPr and three or four sugar-specific proteins or domains (EIIA, EIIB, EIIC, and sometimes EIID). The PTS components EI, HPr, EIIA and EIIB form a phosphorylation cascade with PEP as phosphoryl donor. Phosphorylation occurs at histidyl or cysteyl residues with HPr being phosphorylated at His-15. P EIIB transfers its phosphoryl group to the carbohydrate bound to the corresponding membrane-integrated EIIC and the resulting P-sugar is subsequently released into the cytoplasm. The main function of the PTS is sugar transport, but it is also implicated in numerous regulatory processes. In Gram-positive organisms, PEP- and ATP-dependent phosphorylation of $\mathrm{HPr}$ at His-15 and Ser-46, respectively, are important for the regulatory PTS functions.

Carbon Metabolism and Its Regulation in Lactobacillus casei

\section{Some Carbohydrate Transport Systems of $L$. casei}

\section{PTS Transporters}

L. casei strains contain the genes for the general PTS components EI and HPr [Bettenbrock et al., 1999; Viana et al., 2000] and a large number of operons encoding sugar-specific PTS proteins, many of which belong to the mannose class PTS. A few of them have been characterized in strain BL23. They include the mannose/glucose PTS [Yebra et al., 2006], a PTS transporting fructose molecules derived from the degradation of the fructose polymer levan (PTS ${ }^{\text {Lev}}$ ) [Mazé et al., 2004], a PTS assumed to transport an ester derivative of a sugar, as the corresponding operon contains a gene coding for a sugar-acetyl esterase (PTS ${ }^{\mathrm{Esu}}$ ) [Yebra et al., 2004], and a sorbose-specific PTS containing in its operon also the sorbitol-6-P dehydrogenase-encoding sorF gene [Yebra et al., 2000]. The PTS $^{\text {Sor }}$ and PTS ${ }^{\text {Esu }}$ seem to be absent from strain ATCC334. In contrast, both strains contain a sorF-like gene encoding another sorbitol-6-P dehydrogenase (86\% sequence identity to SorF) [Yebra and Pérez-Martínez, 2002]. While expression of sorF in BL23 was induced by growth on sorbose and glucitol (sorbitol), that of the sorF homologue was induced only by growth on glucitol. It was therefore called $g u t F$ and predicted to play a role in PTS-catalyzed glucitol uptake [Yebra and PérezMartínez, 2002]. Genome sequencing revealed that in ATCC334 and BL23 gutF is located at the beginning of an operon resembling the srl operon of Escherichia coli, which encodes a sorbitol-specific PTS (PTS ${ }^{\mathrm{Gut}}$ ). The $L$. caseigut operon contains genes homologues to E. coli srlD (gutF), srlR, gutM and srlAEB. They encode the sorbitol6-P dehydrogenase GutF, a repressor and a transcription activator [Yamada and Saier, Jr., 1988], and the glucitolspecific PTS components, respectively.

Only strain BL23 is able to metabolize sorbose. In fact, this strain contains a second operon encoding a sorboselike PTS, which is located close to the first sor operon. The second sor operon begins with a third sorF-like gene followed by sor $A B C D$ homologues. The two sor operons (sor and sor2) are only separated by the sorRE genes (divergently oriented to each sor operon). They encode a transcription regulator and a sorbose-1-P reductase, respectively, which might be operative for both sor operons. Interestingly, the sor 2 operon contains behind sorD2 two genes coding for the proteins catalyzing the phosphorylation of dihydroxyacetone (dhaL and dhaK) [Gutknecht et al., 2001]. In E. coli, phosphorylation of EIIA ${ }^{\text {Dha }}$ requires PEP, EI and HPr. P $\sim$ EIIA $^{\text {Dha }}$ in turn transfers its phosphopryl group to ADP bound to DhaL and the resulting

J Mol Microbiol Biotechnol 2007;12:20-32 
ATP:DhaL phosphorylates dihydroxyacetone fixed to DhaK [Bächler et al., 2005]. PEP therefore functions as original phosphoryl donor for dihydroxyacetone phosphorylation. The dha operon of Gram-positive organisms usually contains in addition to dhaKL the gene for the EIIA $^{\text {Dha }}$, but no genes encoding paralogues of EI, HPr or other PTS proteins. Such a system is indeed present in strains BL23 and ATCC334 (dhaKLMS1). The co-localization of the BL23 dhaLK2 genes (inverted gene order compared to dhaKL1) in an operon encoding a complete mannose class PTS is therefore unusual. The true substrates are known neither for the PTS ${ }^{\text {Sor2 }}$ nor for DhaK2/ DhaL2. However, the gene organization suggests that in strain BL23 EIIA ${ }^{\text {Sor2 }}$ might function as phosphoryl donor for the substrate of the PTS and of DhaK2/DhaL2.

The regulation of the dha operon in certain Grampositive organisms is also unusual. DhaS, a TetR-like protein, functions as transcription activator of the Lactococcus lactis dha operon [Christen et al., 2006]. When complexed with dihydroxyacetone, DhaQ, a paralogue of the dihydroxyacetone-binding protein DhaK, functions as co-activator of DhaS. The L. casei dhal operon contains a fourth gene (dhaS1) encoding a homologue of the $L$. lactis TetR-like transcription activator, but misses a dhaQ homologue. It is therefore tempting to assume that in $L$. casei DhaK itself interacts with DhaS when dihydroxyacetone is present.

The lactose-specific PTS of L. casei has been intensively studied. It consists of the lacTEGF genes and encodes a PRD (PTS regulation domain)-containing antiterminator, an EIIBC, a 6-P- $\beta$-galactosidase and an EIIA, respectively. The lac operon was first studied in $L$. casei strain $64 \mathrm{H}$, where it is located on a plasmid [Alpert and Chassy, 1988, 1990; Alpert and Siebers, 1997; Porter and Chassy, 1988], while the lacTEGF operon of strain BL23 is present on the chromosome [Gosalbes et al., 1999]. Nevertheless, homologous lactose-specific proteins of the two strains exhibit about $98 \%$ sequence identity. A lac operon seems to be absent from the chromosome of strain ATCC334 and a plasmid containing the lacTEGF genes has so far not been reported. However, this strain has a lactose-proficient phenotype, suggesting the presence of additional lactose metabolic genes.

The specificity of several PTS can be predicted from sequence comparisons and the localization in an operon with distinct metabolic genes. This is the case for the mannitol- and fructose-specific PTSs. The presumed mannitol-specific PTS operon is composed of the four genes mtlARFD, which encode an EIICB, a PRD-containing transcription activator, an EIIA and a mannitol-1-P dehydrogenase, respectively, as well as the est $B$ gene encoding an arylesterase, which is identical to EstB from $L$. casei LILA [Fenster et al., 2003]. ATCC334 and BL23 contain two presumed fru operons. The frul operon is composed of the three genes fruRKA, which encode a transcription regulator of the DeoR family, a 1-P-fructokinase and an EIIABC, respectively. The fru2 operon only contains the fruKA genes, while a divergently oriented gene (fruR2) encoding a sugar-P-controlled transcription regulator precedes fru2. Interestingly, fruA2 encodes an EII with the domain order BCA instead of $\mathrm{ABC}$. While the domain order $\mathrm{ABC}$ is characteristic of fructose-specific EIIs, BCA is typical for sucrose- and aryl- $\beta$-glucosidespecific EIIs [Deutscher et al., 2002]. Nevertheless, the fruA2-encoded EIIBCA ${ }^{\text {Fru }}$ probably also phosphorylates fructose at the 1-position, since fruK2 codes for a 1-Pfructokinase. Genes resembling fruR2 and fruKA2 are identically organized in Lactobacillus johnsonii (LJ0144LJ0146) [J. Deutscher, unpubl. observation].

Genetic studies provided evidence for the presence of a galactose-specific PTS in strain $64 \mathrm{H}$ [Bettenbrock et al., 1999]. Intracellular galactose-6-P formed either by PTScatalyzed galactose uptake and phosphorylation or resulting from $6-\mathrm{P}-\beta$-galactosidase-catalyzed hydrolysis of lactose-6-P is metabolized via the tagatose-6-P pathway. The genes encoding the enzymes of the tagatose-6-P pathway in strains ATCC 334 and BL23 (galactose-6-P isomerase $\mathrm{a}$ and $\mathrm{b}$, tagatose- $6-\mathrm{P}$ kinase and tagatose-1,6bisphosphate aldolase) are indeed organized in an operon, which is preceded by the lacR gene (encodes a DeoRtype repressor). The PTS, which transports galactose, has so far not been identified. In summary, L. casei strains seem to harbor a vast assortment of PTS transporters, but significant differences (deletions/insertions) exist for various strains.

\section{Non-PTS Transporters}

Galactose can also be taken up via a non-PTS transporter. In this case, intracellular galactose becomes phosphorylated at the C-1 position by ATP and galactokinase and galactose-1-P is metabolized via the Leloir pathway. The genes of the Leloir pathway (galKETRM, encoding the enzymes galactokinase, UDP-glucose 4-epimerase, galactose-1-P uridylyltransferase, a LacI-type repressor and mutarotase, respectively) have first been identified in strain $64 \mathrm{H}$ [Bettenbrock and Alpert, 1998], and nearly identical genes are present in strains ATCC334 and BL23. Several genes encoding transport proteins with about $40 \%$ similarity to the $E$. coli galactose $/ \mathrm{H}^{+}$symporter GalP are also present in strains ATCC334 and BL23. 
Other non-PTS transport systems present in ATCC334 and BL23 include the glycerol and ribose transporters. Glycerol is taken up via facilitated diffusion catalyzed by the glycerol facilitator GlpF. Glycerol kinase (GlpK) converts intracellular glycerol into glycerol-3-P, which is not a substrate for GlpF and therefore remains entrapped in the cell. The $g l p$ operon of $L$. casei is composed of the genes $g l p K O F$, with $g l p O$ encoding a glycerol-3-P dehydrogenase. Interestingly, while strain ATCC334 possesses an intact $g l p O$ gene, the one in BL23 contains a frameshift mutation introducing a stop codon at position 134. This mutation explains why strain BL23 is not able to utilize glycerol. Surprisingly, preliminary experiments suggest that strain ATCC334, in which all glp genes are intact, is also not able to efficiently metabolize glycerol [V. Monedero, unpubl. results]. In contrast, a L. rhamnosus strain containing a very similar $g l p K O F$ operon $(83-92 \%$ identity at the protein level) can utilize glycerol as the sole carbon source [Alvarez et al., 2004].

Ribose is taken up via an ATP-binding cassette (ABC) transport system. The ribose operon is composed of six genes beginning with $r b s R$ encoding a LacI-type repressor. It is followed by the $r b s D A C B K$ genes encoding a ribose mutarotase, a nucleotide-binding protein, a membrane-spanning permease, a ribose-binding protein and ribokinase, respectively.

Maltose is also taken up via an $\mathrm{ABC}$ transport system. The mal operon of strain BL23 is composed of 10 genes encoding a LacI-type repressor (malR), a 1,6- $\alpha$-glucosidase (malL), a neopullulanase (maltogenic amylase) (nplT), a maltose phosphorylase (mapA), a $\beta$-P-glucomutase $(\operatorname{pgmA})$, an ATP-binding protein ( $\mathrm{malK})$, a glucan $1,6-\alpha-$ glucosidase (dexB), a maltose-binding protein (malE) and two membrane-spanning transport proteins (malF and malG). A relation of this operon to maltose metabolism was established by constructing a mapA mutant, which had lost the capacity to grow on maltose and maltotriose [V. Monedero, unpubl. results]. Interestingly, a deletion of a 6.3-kb DNA fragment seems to have occurred in strain ATCC334 leading to the excision of the $3^{\prime}$ half of malK and the entire four following genes (dexB and malEFG). As a consequence, ATCC334 is not able to utilize maltose and maltotriose [V. Monedero, unpubl. results]. Surprisingly, a second operon encoding homologues of MalK, MalF, MalG and MalE is located in both strains about $1.2 \mathrm{~kb}$ downstream of the (entire or truncated) maltose operon and oriented in the opposite direction. The second mal operon possibly transports higher oligomers of maltose.

Interestingly, strain BL23, but not ATCC334, is able to slowly utilize myo-inositol. This capacity is unusual for lactobacilli and is probably due to the insertion of a $12.8-\mathrm{kb}$ DNA fragment in the genome of strain BL23. The inserted DNA contains 11 genes (iolR, iolT, $m m s A$, iolB, iolC, iolD, idh1, idh2, iolE, iolJ and iolK), which encode the repressor IolR and the enzymes catalyzing the transport of myo-inositol (IolT) and its degradation to dihydroxyacetone-P and acetyl-CoA [Yoshida et al., 2004].

\section{CcpA/P-Ser-HPr-Mediated CCR}

\section{The Co-Repressor P-Ser-HPr}

CCR in L. casei follows mechanisms similar to those established in Bacillus subtilis. Sequencing of the L. casei BL23 genome revealed that, with the exception of $\mathrm{crh}$, this organism possesses all genes encoding the components of the PTS/catabolite control protein A (CcpA) CCR pathway: an ATP-dependent HPr kinase/phosphorylase (HprK/P) [Galinier et al., 1998] activated by fructose-1,6bisphosphate (FBP), the HPr protein with a phosphorylatable serine [Viana et al., 2000] and CcpA [Henkin et al., 1991], a member of the LacI-GalR repressor family. A rapid increase in the intracellular concentration of glycolytic intermediates, such as FBP, during the utilization of readily metabolizable sugars is supposed to provide the signal for CCR [Mijakovic et al., 2002]. FBP activates the kinase function of HprK/P, which phosphorylates HPr at a conserved serine residue normally located at position 46, and P-Ser-HPr functions as cofactor for CcpA [Fujita et al., 1995]. The CcpA/P-Ser-HPr complex binds to a 14bp palindromic sequence on the DNA called cre (catabolite responsive element) [Schumacher et al., 2004] and modulates the expression of CCR-regulated genes.

The first crystal structure of an $\mathrm{HprK} / \mathrm{P}$ was obtained for the L. casei BL23 enzyme (truncated active form without the N-terminal domain) [Fieulaine et al., 2001]. It is a homo-hexameric enzyme and the monomer structure resembles that of adenylate kinases and PEP carboxykinases [Galinier et al., 2002; Russell et al., 2002]. The X-ray structure of the whole Staphylococcus xylosus enzyme revealed that the $\mathrm{N}$-terminal domain has structural similarities to the enzyme-I of the PTS and the uridyl-binding region of MurF [Marquez et al., 2002]. The N-terminal domain is, however, dispensable for $\mathrm{HprK} / \mathrm{P}$ function and truncated L. casei HprK/P lacking the first $127 \mathrm{~N}$ terminal amino acids exhibits the same in vitro activity and regulation as the wild-type protein. $\mathrm{HprK} / \mathrm{P}$ is a bifunctional enzyme able to phosphorylate and dephosphorylate HPr [Kravanja et al., 1999]. The kinase function of the L. casei and B. subtilis enzymes is allosteri- 
cally activated by FBP, whereas the enzymes from $S$. xylosus and E. faecalis are regulated by the ATP levels, suggesting that the regulation of $\mathrm{HprK} / \mathrm{P}$ has been adapted to specific physiologic requirements of the different microorganisms. Pi inhibits the kinase function of HprK/ $\mathrm{P}$ and is necessary for P-Ser-HPr dephosphorylation and therefore links P-Ser-HPr formation to the cell metabolic state, as Pi levels increase upon sugar depletion. P-SerHPr dephosphorylation by HprK/P is a phosphorylase reaction with Pi serving as substrate (phospho-phosphorolysis) and pyrophosphate (PPi) and $\mathrm{HPr}$ being the products [Mijakovic et al., 2002]. The phosphorylase reaction is reversible and $\mathrm{HprK} / \mathrm{P}$ can use $\mathrm{PPi}$ as phosphate donor for HPr phosphorylation. It has been postulated that HPrK/P evolved from ancient small substrate-phosphorylating kinases using oligo-phosphates as phosphate donor. In order to catalyze P-Ser-HPr dephosphorylation in response to Pi levels, HprK/P might have retained the ability to use PPi even after it had acquired the capacity to use ATP for HPr phosphorylation.

Several $L$. casei mutant $\mathrm{HprK} / \mathrm{Ps}$ have been isolated displaying normal kinase, but almost no phosphorylase activity [Monedero et al., 2001b]. The mutations affect two different sites: the ATP-binding Walker-A motif (mutations G160S and E163K) and a conserved region called central loop (mutations V267F, G270E, G270R and N272I), which interacts with the Walker-A motif of an adjacent subunit in the hexamer. During P-Ser-HPr dephosphorylation, a Pi molecule occupies the same place as the $\beta$ phosphate of ATP bound to the Walker-A motif. The above mutations probably affect Pi binding without having major effects on ATP binding. Synthesis of the mutant HprK/Ps in B. subtilis caused permanent CCR of a $x y n P^{\prime}$ lacZ fusion. The $h p r K V 267 \mathrm{~F}$ allele had a very strong effect and its expression in B. subtilis led to 'permanent' phosphorylation of HPr at Ser-46. About $95 \%$ of the HPr was transformed into P-Ser-HPr in the absence of a repressing sugar compared to less than $5 \%$ in a wild-type strain. 'Permanent' HPr phosphorylation also occurred when the $h p r K V 267 \mathrm{~F}$ allele was introduced into L. casei. However, allelic replacement was possible only in a $c p A$ background suggesting that P-Ser-HPr accumulation in the presence of functional CcpA causes severe growth defects [V. Monedero and J. Deutscher, unpubl. results].

Evidence that $L$. casei P-Ser-HPr is implicated in CCR was obtained by studying mutants in which Ser-46 was replaced with different amino acids. A L. casei ptsH1 mutant (Ser-46 replaced with the non-phosphorylatable alanine) had lost CCR of reporter activities, such as $\mathrm{N}$-acetylglucosaminidase, and displayed a short diauxic lag phase when grown in mixtures of glucose plus lactose [Viana et al., 2000]. The $p t s H 2$ mutation (Ser- 46 threonine replacement) diminished CCR only slightly. HprK/P slowly phosphorylates HPrS46T and poor dephosphorylation might lead to significant amounts of P-Thr-HPr, which could be responsible for the observed CCR. Similar to hprKV267F, a mutant expressing HPrS46D, which structurally resembles P-Ser-HPr, could not be obtained for $L$. casei. These results suggest that in the presence of wildtype CcpA HPrS46D or elevated amounts of P-Ser-HPr have detrimental effects on $L$. casei growth. The ptsH3 mutation (HPrI47T) was originally isolated in Streptococcus salivarius and provided an HPr exhibiting normal HprK/P-mediated phosphorylation, but probably altered CcpA interaction [Gauthier et al., 1997]. This mutation prevented CCR in L. casei, but also caused overexpression of the lev operon [Mazé et al., 2004]. L. casei HPrI47T is a poor substrate for in vitro PEP- and ATP-dependent phosphorylation. In addition, $\mathrm{P} \sim$ His-HPrI47T transfers its phosphoryl group only slowly to EIIA ${ }^{\text {Lev }}$, which is partly responsible for lev operon deregulation (see chapter 'P $\sim$ His-HPr-Mediated CCR'). This result demonstrates that the CCR-defective phenotype of a L. casei ptsH3 strain is not only due to weakened P-Ser-HPr/ CcpA interaction.

Mutations in the genes coding for the PTS responsible for the transport of glucose and mannose (PTS ${ }^{\mathrm{Man}}$ ) also prevented CCR. Mutants unable to transport glucose via the $\mathrm{PTS}^{\mathrm{Man}}$ displayed elevated $\mathrm{P} \sim \mathrm{His}-\mathrm{HPr}$ levels and no detectable P-Ser-HPr, which explains their CCR-negative phenotype [Yebra et al., 2006].

\section{The Catabolite Repressor CcpA}

CcpA is a regulatory protein belonging to the LacIGalR family of transcriptional regulators and contains an $\mathrm{N}$-terminal helix-turn-helix DNA-binding domain and a C-terminal co-repressor interaction domain resembling periplasmic binding proteins. The $L$. casei $c c p A$ gene is monocistronic and located downstream of $p e p Q$, which is divergently transcribed to $c c p A$ and encodes a prolylaminopeptidase. The $c c p A$-pepQ gene order is conserved in all sequenced members of the Lactobacillales group of firmicutes (except Streptococcus pneumoniae), including lactobacilli, streptococci, lactococci, enterococci, Leuconostoc and oenococci. Contrary to ccpA from S. xylosus and Lactobacillus pentosus, expression of L. casei ccpA is not affected by the presence of a carbon source [Monedero et al., 1997]. The implication of $L$. casei CcpA in CCR was proven by the construction of a $c c p A$ knockout strain, which had lost CCR and diauxic growth [Monedero et al., 
1997]. Synthesis of L. casei CcpA in a B. subtilis ccpA mutant partially restored CCR of a gntR $R K^{\prime}$-lacZ fusion. However, although they share $53 \%$ sequence identity, L. casei CcpA could not replace the $B$. megaterium protein in CCR of a $x y l A^{\prime}$-lacZ fusion [Mahr et al., 2002]. The lack of complementation is the consequence of a 5 -fold lower affinity of L. casei CcpA for B. megaterium P-Ser-HPr compared to L. casei P-Ser-HPr. Some L casei ccpA point mutations caused a slightly different phenotype when compared to the equivalent mutations in $B$. megaterium ccpA [Esteban et al., 2004]. For example, the S80L and T307I mutations, which in B. megaterium led to 'constitutive' CCR, caused a hyper-repressed phenotype in $L$. casei grown in the presence of glucose, whereas they had no major effect in the absence of a repressing sugar. The two mutations affect the cofactor-binding domain and probably strengthen the interaction between co-repressor and repressor.

\section{The Catabolite Response Elements cre}

Numerous cre sites, the binding sites for CcpA/P-Ser$\mathrm{HPr}$, can be found in the promoter region of CCR-sensitive $L$. casei genes. They include the operons encoding the proteins for the utilization of lactose, sorbose, sorbitol, maltose, inositol and the lev and esu operons as well as the gene encoding $\mathrm{N}$-acetylglucosaminidase [Senba et al., 2000] (table 1). cre sites can be identified not only in operons related to sugar utilization but also in operons containing glycolytic genes, such as $p f k-p y k$. Although these cre sites deviate from the consensus sequence, they were recognized by the CcpA/P-Ser-HPr complex in vitro, suggesting that in L. casei CcpA is also participating in the regulation of central metabolic genes [Viana et al., 2005].

\section{P His-HPr-Mediated CCR}

CCR can also be mediated by $\mathrm{P} \sim \mathrm{His}-\mathrm{HPr}$, which transfers its phosphoryl group not only to EIIAs of the PTS, but also to several non-PTS proteins. Polypeptides phosphorylated by $\mathrm{P} \sim \mathrm{His}-\mathrm{HPr}$ in Gram-positive bacteria include GlpK and numerous PRD-containing antiterminators and transcription activators. In all cases, phosphorylation by $\mathrm{P} \sim \mathrm{His}-\mathrm{HPr}$ leads to an increase of the catalytic activity.

\section{Regulation of Glycerol Kinase}

As mentioned before, both partially sequenced L. casei strains possess a $g l p K O F$ operon. The $g l p O$ gene of strain BL23 contains a frameshift mutation creating a stop co-
Table 1. cre sites present in the promoter region of some L. casei genes

\begin{tabular}{ll}
\hline Gene & cre sequence \\
\hline lacT (lac operon antiterminator) & ATAAAACGTTTACA \\
sorF (sorbitol-6P dehydrogenase) & TGTAACCGGTTACA \\
levA (EIIA ${ }^{\text {Lev }}$ ) & TGTAAGCGTTAACC \\
esuA (EIIA ${ }^{\text {Esu }}$ ) & TGTAAACGTATGAA \\
iolT (inositol transporter) & ATCGTGCGAATTCA \\
malL ( $\alpha-1,6$-glucosidase) & TGCGTGCGCTTTCA \\
gutF (sorbitol-6P dehydrogenase) & CGTAAACGCTACCA \\
nagA (N-acetylglucosaminidase) & TGAAAGCGATTCCA \\
pfk (phosphofructokinase) & TGTTCTCATTTTCA \\
& TGTTTCTGAAAAGT \\
\hline B. subtilis consensus & TGWNANCGNTNWCA \\
\hline
\end{tabular}

don at position 134, while ATCC334 contains intact $g l p$ genes. Nevertheless, both strains are not able to efficiently utilize glycerol [V. Monedero, unpubl. results]. GlpK of Gram-positive organisms has been shown to require phosphorylation by $\mathrm{P} \sim \mathrm{His}-\mathrm{HPr}$ to be active and mutations in the general PTS proteins EI or HPr therefore prevent the metabolism of glycerol [Beijer and Rutberg, 1992; GonzyTréboul et al., 1991; Reizer et al., 1984b]. However, GlpK of BL23 and ATCC334 contains a well-conserved site for $\mathrm{P} \sim$ His-HPr-mediated phosphorylation (His-233), which is surrounded by three aromatic amino acids (-Y-H-F-F-) similar to the phosphorylation site in GlpK from other Gram-positive organisms [Charrier et al., 1997; Darbon et al., 2002]. It is therefore likely that in the absence of a rapidly metabolizable PTS sugar GlpK of the two L. casei strains will be activated via phosphorylation by $\mathrm{P} \sim$ HisHPr. The inability of strain ATCC334 to utilize glycerol must therefore be related to another defect. As already mentioned, $L$. rhamnosus possesses a very similar $g l p K O F$ operon and utilizes glycerol as the sole carbon source.

Similar to L. rhamnosus, L. casei ATCC334 and BL23 possess a second $g l p K$. In L. rhamnosus, glpK 2 is truncated at the C-terminus (439 aa instead of the usually 505) and was called $g y k A$ [Alvarez et al., 2004]. In L. casei, $g y k A$ is monocistronic and located in front of the fru 2 operon. GykA of L. rhamnosus and L. casei exhibit 90\% sequence identity, while L. casei GlpK and GykA show only 78\% sequence identity. The function of GykA is unknown, but in both organisms it contains a $\mathrm{P} \sim \mathrm{His}-\mathrm{HPr}$ phosphorylation site in position 231 identical to that in GlpK (-Y-H-FF-). Nevertheless, in L. rhamnosus, gykA was not induced during growth on glycerol [Alvarez et al., 2004].

J Mol Microbiol Biotechnol 2007;12:20-32 
Regulation of PRD-Containing Antiterminators

L. casei possesses a few antiterminators containing two PRDs. These antiterminators bind to specific sites (RAT sequence) on the nascent mRNA and prevent the formation of a terminator located in front of the transcription units, thereby allowing transcription of the fulllength mRNA [Deutscher et al., 2002]. One of the L. casei antiterminators, LacT, has been studied in more detail. LacT controls the expression of the lacTEGF operon described before and is present in strains $64 \mathrm{H}$ (plasmid-encoded) and BL23 (chromosomal) [Alpert and Siebers, 1997; Gosalbes et al., 1999]. Similar to LicT of B. subtilis [Tortosa et al., 2001], LacT seems to be regulated by two PTS-mediated phosphorylation reactions exerting antagonistic effects on its activity. Phosphorylation at His-101 in PRD1 requires $\mathrm{P} \sim \mathrm{EIIB}^{\mathrm{Lac}}$ and lowers the antitermination activity of LacT. Consequently, inactivation of EIIA $^{\mathrm{Lac}}$ or EIIB ${ }^{\mathrm{Lac}}$ [Gosalbes et al., 1999] as well as replacement of His-101 in LacT with an alanine leads to constitutive expression of the lac operon, while replacement with an Asp, which mimics phosphorylation owing to its negative charge, prevents lac operon expression [Gosalbes et al., 2002]. Phosphorylation at His-101 occurs when lactose is absent from the growth medium. If lactose is present, the phosphoryl group of $\mathrm{P} \sim \mathrm{EIIB}^{\mathrm{Lac}}$ will mainly be used for the phosphorylation of lactose and LacT will primarily be present in its unphosphorylated active form. The poor phosphorylation of LacT at His-101 in the presence of lactose therefore leads to the induction of the lac operon. Phosphorylation at either His-210 or His-273 in PRD2 is probably mediated by $\mathrm{P} \sim \mathrm{His}-\mathrm{HPr}$ and stimulates LacT activity. For LicT of B. subtilis it has been shown that during growth on a rapidly metabolizable PTS sugar $\mathrm{P} \sim \mathrm{His}-\mathrm{HPr}$-mediated phosphorylation in PRD2 is diminished leading to low LicT activity. The absence of phosphorylation of PRD2 of some antiterminators represents a CcpA-independent CCR mechanism. Genetic studies in a $c c p A^{-}$background suggest that this mechanism is also operative for LacT of $L$. case $i$ [Gosalbes et al., 2002].

L. casei strains ATCC 334 and BL23 contain genes for two other PRD-containing antiterminators. One is located next to, the other within an operon encoding a $\beta$-glucoside-specific EIIBCA strongly resembling BglP of $B$. subtilis and a 6-P- $\beta$-glucosidase resembling $\mathrm{BglH}$.

\section{Regulation of PRD-Containing Transcription \\ Activators}

PRDs control the activity of not only antiterminators, but also of certain transcription activators, which possess two PRDs, an EIIB ${ }^{\text {Gat }}$ domain and an EIIA domain of either the mannose or mannitol/fructose class PTS. They therefore contain up to six potential PTS phosphorylation sites (some transcription activators possesses a truncated PRD2 with only one phosphorylatable His) [Deutscher et al., 2002]. Two classes of PRD-containing transcription activators exist. One has a DNA-binding domain resembling DeoR-like transcription regulators, the other has an N-terminal helix-turn-helix motif followed by an NtrC central domain with a Walker-A motif located around position 150.

L. casei BL23 contains two transcription activators of the latter type, one of which (LevR) is present also in ATCC334 and has been extensively studied [Mazé et al., 2004]. LevR controls the expression of the lev operon, which is composed of the four genes encoding the PTS ${ }^{\mathrm{Lev}}$ components (levABCD), levX of unknown function and, in strain ATCC334, a sacC homologue, which encodes an extracellular levanase degrading the fructose polymer levan. The $s a c C$ gene is missing in strain BL23, but neighboring levX and $s a c C$ homologues were found in another L. casei strain (GenBank accession No. AB185852). The $\mathrm{PTS}^{\mathrm{Lev}}$ transports fructose and mannose. The levR gene is located in front of the lev operon and is oriented in the opposite direction [Mazé et al., 2004]. Similar to PRDcontaining antiterminators, the activity of LevR is regulated by two PTS-mediated phosphorylation reactions exerting antagonistic effects on LevR function. The two sites of phosphorylation are His-488 in the EIIA ${ }^{\text {Man }}$ domain and His-776 in the truncated PRD2. Phosphorylation in PRD2 is mediated by $\mathrm{P} \sim \mathrm{EIIB}^{\mathrm{Lev}}$ and leads to the inactivation of LevR. Disruption of levB therefore causes constitutive expression of the lev operon [Mazé et al., 2004]. This regulatory mechanism is used for the induction of the lev operon. If a substrate of the PTS ${ }^{\mathrm{Lev}}$ is present, $\mathrm{P} \sim \mathrm{EIIB}^{\mathrm{Lev}}$ will preferentially transfer its phosphoryl group to the carbohydrate and LevR will be barely phosphorylated in PRD2 and therefore be active. In contrast, phosphorylation at His- 488 in the EIIA ${ }^{\text {Man }}$ domain stimulates LevR activity and its absence serves as a CcpA-independent CCR mechanism. The P $\sim$ His-HPr-mediated phosphorylation is probably prevented when a rapidly metabolizable PTS sugar is taken up. In agreement with this concept, a levB ptsI double mutant, in which LevR is phosphorylated neither in PRD2 nor in the EIIA Man domain, exhibited significantly lower LevR activity than a levB single mutant [Mazé et al., 2004].

The well-studied LevR of B. subtilis [Deutscher et al., 2002] requires RpoN $\left(\sigma^{54}\right)$ to carry out its transcription activation function. Interestingly, L. casei LevR remained 
active when rpoN was disrupted [Mazé et al., 2004]. In fact, $L$. casei LevR misses the -G-(A,S)-F-T-G-A- sequence in the NtrC-like central domain, which is required for the interaction with RpoN. In addition, the lev operon is not preceded by a $-12,-24$ promoter, which is recognized by the RNA polymerase/RpoN holoenzyme. Moreover, strain ATCC334 contains the lev operon, but no rpoN gene. Strain BL23, in contrast, possesses an rpoN gene located behind esuR, which encodes the second LevR-like transcription activator of L. casei BL23 [Yebra et al., 2004]. EsuR controls the expression of the esu $A B C D E$ operon, which encodes the four components of a mannose class PTS and a sugar-acetyl esterase. If either $r p o N$ or $e s u R$ were disrupted in strain BL23, expression of the esu operon was strongly diminished [Yebra et al., 2004]. Expression of the esu operon therefore depends on RpoN. The esu operon, which is absent from strain ATCC334, is preceded by a typical -12,-24 promoter, and EsuR contains the RpoN-binding motif -G-S-F-T-G-A-.

The two L. casei strains ATCC334 and BL23 also contain DeoR-type PRD containing transcription activators. One of them is encoded by the already mentioned $m t l R$ gene from the $m t l$ operon, which also encodes the components of a mannitol-specific PTS (mtlAF) and mannitol1-P dehydrogenase (mtlD). MtlR of Geobacillus stearothermophilus has been extensively studied [Henstra et al., 1999, 2000] and its phosphorylation sites have been identified. MtlR of L. casei contains equivalent phosphorylatable histidines and is probably regulated by the PTS similar to G. stearothermophilus MtlR. Both partially sequenced strains contain three other MtlR-like transcription activators, which have so far not been characterized.

\section{Inducer Exclusion}

The presence of readily metabolizable sugars can prevent the uptake of less preferred carbon sources. Blocking the transport step of a sugar prevents the formation of the corresponding intracellular inducer and the mechanism was therefore called inducer exclusion. In enterobacteriaceae, inducer exclusion is mediated by dephosphoEIIA $^{\text {Glc }}$, which can interact with different non-PTS permeases and inhibit their activity [Postma et al., 1993]. Early evidence for the participation of proteins of the PTS in inducer exclusion in low-G+C Gram-positive bacteria was obtained with Lactobacillus brevis. Its lactose and glucose permeases were shown to interact with HPrS46D from B. subtilis [Ye and Saier, Jr., 1995a, 1995b]. The $L$. brevis galactose exclusion system including $p t s H$ and the galactose $/ \mathrm{H}^{+}$symporter-encoding galP was reconstituted in B. subtilis [Djordjevic et al., 2001]. Inducer exclusion is not operative in B. subtilis, as the inability of an hprKV267F mutant (accumulates P-Ser-HPr) to grow on minimal medium complemented with various non-PTS sugars did not persist in a $c c p A^{-}$background [Monedero et al., 2001a]. However, when L. brevis galP was expressed in B. subtilis, addition of glucose or synthesis of $L$. brevis HPrS46D inhibited the transport of thiomethyl- $\beta$-D-galactoside (TMG) via GalP. This experiment reconstituted inducer exclusion in an organism that naturally lacks this regulatory phenomenon. With inducer exclusion of nonPTS sugars being absent from $B$. subtilis, $L$. casei became the Gram-positive model organism to study the molecular mechanisms underlying this phenomenon. Experiments with $L$. casei $p t s H 1$ and $h p r K$ mutants provided the first in vivo evidence that P-Ser-HPr participates in inducer exclusion. Glucose, which inhibits maltose transport in a wild-type strain, had no effect on the transport of the disaccharide in the two mutant strains [Dossonnet et al., 2000; Viana et al., 2000]. Maltose exclusion was also not operative in L. casei $p t s \mathrm{H} 2$ and $p t s \mathrm{H} 3$ mutants. Moreover, in a $L$. casei ccpA $h p r K V 267 \mathrm{~F}$ double mutant (accumulates P-Ser-HPr) growth on maltose and maltose uptake were strongly inhibited [V. Monedero and J. Deutscher, unpubl. results]. Similar results were obtained with L. lactis, where a $\mathrm{ptsH} 1$ mutation prevented glucosemediated exclusion of maltose and ribose [Monedero et al., 2001a]. L. casei and L. lactis take up maltose and ribose via $\mathrm{ABC}$ transporters. In E. coli, EIIA ${ }^{\mathrm{Glc}}$ interacts with the ATPase subunit (MalK) of the maltose-specific $\mathrm{ABC}$ transporter and inhibits its activity. It is therefore likely that the $L$. casei MalK homologue might be inhibited by P-Ser-HPr (fig. 1). A sequence comparison of the ATPase subunits of the maltose and ribose ABC-transporters from $L$. casei and L. lactis did not allow the identification of specific target regions for regulation. However, a comparison of E. coli and L. casei MalK showed that they contain very similar N-terminal ATP-binding domains ( $60 \%$ identity), whereas the C-terminal regulatory domain is less conserved (19\% identity) [V. Monedero and J. Deutscher, unpubl. observation]. The poor conservation of the regulatory domain might reflect the interaction with the different effectors EIIA $^{\text {Glc }}$ and P-Ser-HPr. 
Fig. 1. The present model of inducer exclusion in Gram-positive organisms. In L. casei, maltose is taken up via an ABC transport system composed of the maltose-binding protein MalE, the membrane proteins MalF and MalG and the ATP-binding protein $\mathrm{MalK}_{2}$, (present as dimer), while glucose is transported via a mannose class PTS (EIIABCD). When glucose is present, its rapid metabolism raises the concentration of FBP, which stimulates the HprK/P-catalyzed formation of P-Ser-HPr. P-Ser-HPr probably interacts with a component of the $\mathrm{ABC}$ transporter (most likely $\mathrm{MalK}$, as the ATP-binding protein of enterobacteriaceae is inhibited by EIIA ${ }^{\mathrm{Glc}}$ ) and thereby inhibits maltose uptake. In mutants unable to form P-Ser-HPr ( $h p r K$, ptsH1, etc.), maltose transport is no longer inhibited by glucose.

Fig. 2. The mechanism of TMG expulsion in L. casei. TMG is taken up via the lactose-specific PTS and accumulated as TMG6 -P, because the enzyme 6-P- $\beta$-galactosidase cannot hydrolyze the thio-galactoside bond. The presence of glucose or other rapidly metabolizable sugars triggers the two-step expulsion of TMG-6-P: intracellular dephosphorylation followed by the export of TMG. The detailed mechanism is not known. A low MW phosphatase was proposed to catalyze TMG-6-P dephosphorylation and TMG would then be expelled by an unknown exporter or via the lactose-specific PTS. It is also possible that the PTS ${ }^{\mathrm{Lac}}$ catalyzes both dephosphorylation and the subsequent export. In this case, the PTS would run backwards, i.e. EIIB ${ }^{\mathrm{Lac}}$ would accept the phosphoryl group from TMG-6-P, similar as proposed for the so-called transphosphorylation reaction [Rephaeli and Saier, Jr., 1978]. Whatever the detailed mechanism may be, the proposed role of P-Ser-HPr in inducer expulsion could not be confirmed. L. casei mutants unable to form P-Ser-HPr ( $h p r K$, ptsH1, etc.) could still expel TMG as efficiently as the wild-type strain.

\section{Inducer Expulsion}

L. casei can transport and accumulate phosphorylated derivatives of non-metabolizable PTS sugars, such as TMG. Accumulated P-sugars are expelled into the medium when a rapidly metabolized sugar is added. This phenomenon, first observed with TMG in Streptococcus pyogenes [Reizer and Panos, 1980], was designated inducer expulsion by analogy to inducer exclusion. However, inducer expulsion seems to be a detoxification mechanism to expel accumulated toxic non-metabolizable sugar-phosphates rather than a regulatory mechanism for carbon metabolism. Inducer expulsion is an ATP-requiring two-step process, during which the sugar-phosphate is first dephosphorylated and subsequently expelled from the cell (fig. 2). In-
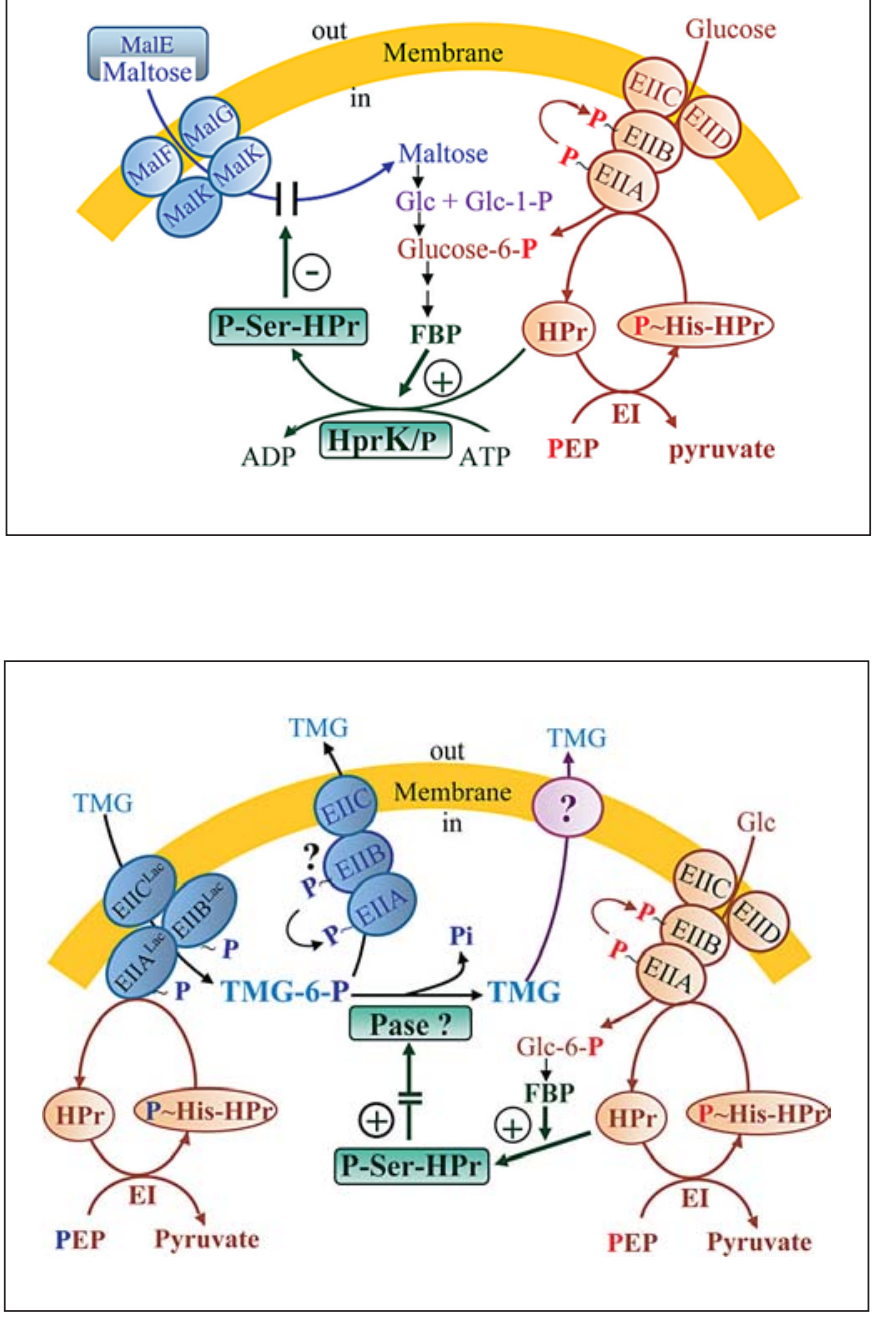

terestingly, conditions which elicit inducer expulsion also lead to the formation of P-Ser-HPr [Reizer et al., 1984a].

Moreover, electroporation of B. subtilis HPrS46D into L. lactis vesicles, which had accumulated TMG-6-P or 2deoxyglucose-6-P, provoked rapid expulsion of the sugars. Electroporation of HPr and different glycolytic intermediates, which induce phosphorylation of HPr by $\mathrm{HprK} / \mathrm{P}$ in the vesicles, had similar effects. The search for specific $L$. lactis sugar-P phosphatases catalyzing the first step of expulsion led to the characterization of two different phosphatases (Pase I and Pase II) [Ye and Saier, Jr., $1995 \mathrm{c}]$. The small Pase II (10 kDa) is membrane-associated, dephosphorylates a wide range of sugar-phosphates and is the only phosphatase allosterically activated by HPrS46D. In agreement with the participation of Pase II 
in inducer expulsion, Pase II-like activities were only detected in bacteria exhibiting inducer expulsion, whereas they were absent from bacteria lacking this phenomenon, such as S. aureus, S. mutans, S. salivarius or B. subtilis [Ye et al., 1996]. With inducer expulsion being absent from the Gram-positive model organism B. subtilis, the L. casei $p t s H 1$ and $h p r K$ mutants provided the first opportunity to test the proposed involvement of P-Ser-HPr in inducer expulsion in vivo. L. casei takes up TMG via $\mathrm{EIICB}^{\mathrm{Lac}}$ and accumulates it as TMG-6-P (fig. 2). The ptsH1 and $h p r K$ mutations had no effect on glucose-induced expulsion of TMG-6-P, indicating that inducer expulsion does not require P-Ser-HPr in this organism [Dossonnet et al., 2000]. Similarly, a L. lactis ptsH1 mutant was not affected in 2-deoxyglucose expulsion, although it expelled TMG at a significantly slower rate [Monedero et al., 2001a]. The reduced TMG expulsion may be caused by an indirect effect. It has been postulated that $\mathrm{EIICB}^{\mathrm{Lac}}$ catalyzes both TMG uptake and TMG-6-P expulsion (fig. 2) [Reizer and Saier, Jr., 1983]. When grown on glucose, the absence of P-SerHPr in the ptsH1 mutant might lead to elevated phosphorylation of EIICB ${ }^{\mathrm{Lac}}$, which might favor TMG transport and phosphorylation rather than its expulsion. In any case, the mechanism of inducer expulsion remains elusive. The characterization of Pase II provided evidence for an implication of P-Ser-HPr in inducer expulsion, whereas in vivo analyses in L. casei and L. lactis argue against it.

\section{Relation between the PTS and Cold Shock Response}

Cold is a physical stress that drastically alters all physico-chemical parameters of a living cell by affecting solute diffusion rates, enzyme kinetics, membrane fluidity and the conformation, flexibility, topology and reciprocal interactions of macromolecules such as DNA, RNA and proteins. Bacteria adapt to a sudden drop in temperature with a drastic reduction of the growth rate as well as a marked reprogramming of protein synthesis. The most strongly cold-induced proteins (Cips) are the small $(7.5 \mathrm{kDa})$ acidic cold shock proteins (Csps) present in most Gram-positive and Gram-negative bacteria. Bacteria usually harbor several Csp homologues, of which only a subset may be cold-inducible, and genetic analysis showed that they play vital roles not only during cold shock [Graumann et al., 1997]. Interestingly, subdomains homologous to Csps have been identified in several important eukaryotic proteins [Weber and Marahiel, 2003]. Determination of the three-dimensional structures in combination with biochemical studies revealed that Csps have a conserved fold that allows binding to singlestranded nucleic acids [Weber and Marahiel, 2003].

The first indication for a relation between the bacterial cold shock response and PTS/CCR components came from studies in B. subtilis [Graumann et al., 1997] and $L$. lactis [Wouters et al., 2000], which showed that HPr is part of the Cips in both organisms and that expression of L. lactis ccpA was cold-inducible. Recently, CCR-relieved L. casei mutants were reported to overproduce a Csp, thus strengthening the suggested link between Csps and PTS/ CCR components [Beaufils et al., 2006]. Furthermore, freeze/thaw experiments with the CCR-relieved mutants grown on glucose revealed a similar survival rate for wild-type, $c c p A$, ptsH2 and $p t s H 3$ strains, whereas the ptsH1 and hprK mutants, which are not able to form PSer-HPr, were 80 -fold more sensitive. Varying the ratio of the different HPr forms ( $\mathrm{HPr}, \mathrm{P} \sim \mathrm{His}-\mathrm{HPr}, \mathrm{P}-\mathrm{Ser}-\mathrm{HPr}$ and doubly phosphorylated $\mathrm{HPr}$ ) by growing the cells on different non-PTS sugars altered the survival rate of the ptsH1 and hprK strains. HPr or one of its phospho-derivatives might therefore bind to or phosphorylate CspA. However, despite additional studies with a ptsI mutant, which cannot form $\mathrm{P} \sim \mathrm{His}-\mathrm{HPr}$, and in vitro experiments aimed to demonstrate phosphorylation or interaction of L. casei CspA with the various HPr forms, it remains obscure how HPr contributes to the resistance to cold and freezing conditions [Beaufils et al., 2006].

The crystal structure of several Csps has been solved [Weber and Marahiel, 2003]. They show a very similar overall fold consisting of five antiparallel $\beta$-sheets that form a $\beta$-barrel structure with surface exposed aromatic and basic residues, which are involved in nucleic acid binding. Interestingly, the crystal structure of the translation initiation factor IF1 is almost identical to that of Csps and the two proteins exert overlapping cellular functions [Weber et al., 2001]. Moreover, Csps and the Cterminal domain of EIIA ${ }^{\text {Glc }}$ have a very similar structure, too [Beaufils et al., 2006]. In contrast to IF1, Csps and EIIA $^{\text {Glc }}$ show also significant sequence similarity and most important, the histidyl residue of EIIA ${ }^{\text {Glc }}$, which is phosphorylated by $\mathrm{P} \sim \mathrm{His}-\mathrm{HPr}$, is conserved in Csps. In both proteins this histidine is located at the end of the third $\beta$-strand of the central $\beta$-sheet. Site-directed mutagenesis experiments showed that this histidine is important for the in vivo and in vitro functions of $E$. coli CspE [Phadtare, 2004; Phadtare et al., 2002a, 2002b]. Interestingly, DNA microarray-based global transcript profiling of $E$. coli showed that several genes encoding PTS EIIcomponents are significantly induced by cold shock [Phadtare, 2004]. In conclusion, there is accumulating 
evidence for a close relationship between PTS and Csps and it is tempting to assume that HPr or one of its P-derivatives interacts with or phosphorylates Csps in vivo.

\section{Acknowledgements}

We thank Noémie Jacques and Karine Correia for technical assistance during genome sequencing of $L$. casei strain BL23.

\section{Note Added in Proof}

The genome sequence of strain ATCC334 has been completed during the preparation of this paper and is available at NCBI under http://www.ncbi.nlm.nih.gov/. Plasmid 1 of strain ATCC334 has also been sequenced. It contains a complete lac operon (lacTEGF), which probably explains the ability of this strain to utilize lactose.

\section{References}

Alpert, C.-A., Chassy, B.M. 1988. Molecular cloning and nucleotide sequence of the factor III ${ }^{\mathrm{Lac}}$ gene of Lactobacillus casei. Gene 62: 277-288.

Alpert, C.-A., Chassy, B.M. 1990. Molecular cloning and DNA sequence of $l a c E$, the gene encoding the lactose-specific enzyme II of the phosphotransferase system of Lactobacillus casei. Evidence that a cysteine residue is essential for sugar phosphorylation. J Biol Chem 265:22561-22568.

Alpert, C.A., Siebers, U. 1997. The lac operon of Lactobacillus casei contains lacT, a gene coding for a protein of the BglG family of transcriptional antiterminators. J Bacteriol 179: 1555-1562.

-Alvarez, M.d.F., Medina, R., Pasteris, S.E., Strasser de Saad, A.M., Sesma, F. 2004. Glycerol metabolism of Lactobacillus rhamnosus ATCC 7469: Cloning and expression of two glycerol kinase genes. J Mol Microbiol Biotechnol 7:170-181.

- Bächler, C., Flükiger-Brühwiler, K., Schneider, P., Bähler, P., Erni, B. 2005. From ATP as substrate to ADP as coenzyme: Functional evolution of the nucleotide binding subunit of dihydroxyacetone kinase. J Biol Chem 280: 18321-18325.

Beaufils, S., Sauvageot, N., Mazé, A., Laplace, J.M., Auffray, Y., Deutscher, J., Hartke, A. 2006. The cold shock response of Lactobacillus casei: Relation between HPr phosphorylation and resistance to freeze/thaw cycles. J Mol Microbiol Biotechnol, in press.

- Beijer, L., Rutberg, L. 1992. Utilisation of glycerol and glycerol 3-phosphate is differently affected by the phosphotransferase system in Bacillus subtilis. FEMS Microbiol Lett 100: 217-220.

Bettenbrock, K., Alpert, C.-A. 1998. The gal genes for the Leloir pathway of Lactobacillus casei 64H. Appl Environ Microbiol 64:2013-2019.

Bettenbrock, K., Siebers, U., Ehrenreich, P., Alpert, C.-A. 1999. Lactobacillus casei $64 \mathrm{H}$ contains a phosphoenolpyruvate-dependent phosphotransferase system for uptake of galactose, as confirmed by analysis of $\mathrm{ptsH}$ and different gal mutants. J Bacteriol 181:225-230.
Charrier, V., Buckley, E., Parsonage, D., Galinier, A., Darbon, E., Jaquinod, M., Forest, E., Deutscher, J., Claiborne, A. 1997. Cloning and sequencing of two enterococcal $g l p K$ genes and regulation of the encoded glycerol kinases by phosphoenolpyruvate-dependent, phosphotransferase system-catalyzed phosphorylation of a single histidyl residue. J Biol Chem 272:14166-14174.

Christen, S., Srinivas, A., Bahler, P., Zeller, A., Pridmore, D., Bieniossek, C., Baumann, U., Erni B. 2006. Regulation of the dha operon of Lactococcus lactis: A deviation from the rule followed by the TetR family of transcription regulators. J Biol Chem June 7, Epub ahead of print.

Coeuret, V., Guéguen, M., Vernoux, J.P. 2004. Numbers and strains of lactobacilli in some probiotic products. Int J Food Microbiol 97: 147-156.

Darbon, E., Servant, P., Poncet, S., Deutscher, J. 2002. Antitermination by GlpP, catabolite repression via $\mathrm{CcpA}$, and inducer exclusion elicited by $\mathrm{P} \sim \mathrm{GlpK}$ dephosphorylation control Bacillus subtilis glpFK expression. Mol Microbiol 43:1039-1052.

Dellaglio, F., Felis, G.E., Torriani, S. 2002. The status of the species Lactobacillus casei (Orla-Jensen 1916) Hansen and Lessel 1971 and Lactobacillus paracasei Collins et al. 1989. Request for an opinion. Int J Syst Evol Microbiol 52:285-287.

Deutscher, J., Galinier, A., Martin-Verstraete, I. 2002. Carbohydrate uptake and metabolism; in Sonenshein, A.L., Hoch, J.A., Losick, R. (eds): Bacillus subtilis and its closest relatives: From genes to cells. Washington, ASM Press, 2002, pp 129-150.

-Dicks, L.M., Du Plessis, E.M., Dellaglio, F., Lauer, E. 1996. Reclassification of Lactobacillus casei subsp. casei ATCC 393 and Lactobacillus rhamnosus ATCC 15820 as Lactobacillus zeae nom. rev., designation of ATCC 334 as the neotype of L. casei subsp. casei, and rejection of the name Lactobacillus paracasei. Int J Syst Bacteriol 46:337-340.
Djordjevic, G.M., Tchieu, J.H., Saier Jr, M.H. 2001. Genes involved in control of galactose uptake in Lactobacillus brevis and reconstitution of the regulatory system in Bacillus subtilis. J Bacteriol 183:3224-3236.

Dossonnet, V., Monedero, V., Zagorec, M., Galinier, A., Pérez-Martínez, G., Deutscher, J. 2000. Phosphorylation of HPr by the bifunctional HPr kinase/P-Ser-HPr phosphatase from Lactobacillus casei controls catabolite repression and inducer exclusion but not inducer expulsion. J Bacteriol 182:2582-2590.

Esteban, C.D., Mahr, K., Monedero, V., Hillen, W., Pérez-Martínez, G., Titgemeyer, F. 2004 Complementation of a DccpA mutant of Lactobacillus casei with CcpA mutants affected in the DNA- and cofactor-binding domains. Microbiology 150:613-620.

Fenster, K.M., Parkin, K.L., Steele, J.L. 2003. Nucleotide sequencing, purification, and biochemical properties of an arylesterase from Lactobacillus casei LILA. J Dairy Sci 86: 2547-2557.

Fieulaine, S., Morera, S., Poncet, S., Monedero, V., Gueguen-Chaignon, V., Galinier, A., Janin, J., Deutscher, J., Nessler, S. 2001. X-ray structure of HPr kinase: a bacterial protein kinase with a P-loop nucleotide binding domain. EMBO J 20:3917-3927.

Fujita, Y., Miwa, Y., Galinier, A., Deutscher, J. 1995. Specific recognition of the Bacillus subtilis gnt cis-acting catabolite-responsive element by a protein complex formed between CcpA and seryl-phosphorylated HPr. Mol Microbiol 17:953-960.

-Galinier, A., Kravanja, M., Engelmann, R. Hengstenberg, W., Kilhoffer, M.-C., Deutscher, J., Haiech, J. 1998. New protein kinase and protein phosphatase families mediate signal transduction in bacterial catabolite repression. Proc Natl Acad Sci USA 95:18231828.

Galinier, A., Lavergne, J.P., Geourjon, C., Fieulaine, S., Nessler, S., Jault, J.M. 2002. A new family of phosphotransferases with a P-loop motif. J Biol Chem 277:11362-11367. 
Gauthier, M., Brochu, D., Eltis, L.D., Thomas, S., Vadeboncoeur, C. 1997. Replacement of isoleucine-47 by threonine in the HPr protein of Streptococcus salivarius abrogates the preferential metabolism of glucose and fructose over lactose and melibiose but does not prevent the phosphorylation of HPr on serine-46. Mol Microbiol 25:695-705.

-Gonzy-Tréboul, G., de Waard, J.H., Zagorec, M., Postma, P.W. 1991. The glucose permease of the phosphotransferase system of Bacillus subtilis: evidence for $\mathrm{II}^{\mathrm{Glc}}$ and $\mathrm{III}^{\mathrm{Glc}}$ domains. Mol Microbiol 5:1241-1249.

Gosalbes, M.J., Esteban, C.D., Pérez-Martínez, G. 2002. In vivo effect of mutations in the antiterminator LacT in Lactobacillus casei. Microbiology 148:695-702.

-Gosalbes, M.J., Monedero, V., Pérez-Martínez, G. 1999. Elements involved in catabolite repression and substrate induction of the lactose operon in Lactobacillus casei. J Bacteriol 181:3928-3934.

Graumann, P., Wendrich, T.M., Weber, M.H.W., Schröder, K., Marahiel, M.A. 1997. A family of cold shock proteins in Bacillus subtilis is essential for cellular growth and for efficient protein synthesis at optimal and low temperatures. Mol Microbiol 25:741-756.

-Gutknecht, R., Beutler, R., Garcia-Alles, L.F., Baumann, U., Erni, B. 2001. The dihydroxyacetone kinase of Escherichia coli utilizes a phosphoprotein instead of ATP as phosphoryl donor. EMBO J 15:2480-2486.

-Henkin, T.M., Grundy, F.J., Nicholson, W.L., Chambliss, G.H. 1991. Catabolite repression of $\alpha$-amylase gene expression in Bacillus subtilis involves a trans-acting gene product homologous to the Escherichia coli lacI and galR repressors. Mol Microbiol 5:575-584.

-Henstra, S.A., Duurkens, R.H., Robillard, G.T. 2000. Multiple phosphorylation events regulate the activity of the mannitol transcriptional regulator MtlR of the Bacillus stearothermophilus phosphoenolpyruvate-dependent mannitol phosphotransferase system. J Biol Chem 275:7037-7044.

Henstra, S.A., Tuinhof, M., Duurkens, R.H., Robillard, G.T. 1999. The Bacillus stearothermophilus mannitol regulator, MtlR, of the phosphotransferase system. A DNA-binding protein, regulated by $\mathrm{HPr}$ and $\mathrm{IICB}^{\mathrm{mtl}}$-dependent phosphorylation. J Biol Chem 274: 4754-4763.

Klaenhammer, T., Altermann, E., Arigoni, F., Bolotin, A., Breidt, F., Broadbent, J., Cano, R., Chaillou, S., Deutscher, J., Gasson, M., van de Guchte, M., Guzzo, J., Hartke, A., Hawkins, T., Hols, P., Hutkins, R., Kleerebezem, M., Kok, J., Kuipers, O., Lubbers, M., Maguin, E., McKay, L., Mills, D., Nauta, A., Overbeek, R., Pel, H., Pridmore, D., Saier, M., van Sinderen, D., Sorokin, A., Steele, J., O’Sullivan, D., de Vos, W., Weimer, B., Zagorec, M., Siezen, R. 2002. Discovering lactic acid bacteria by genomics. Antonie Van Leeuwenhoek 82:29-58.
Kravanja, M., Engelmann, R., Dossonnet, V., Blüggel, M., Meyer, H.E., Frank, R., Galinier, A., Deutscher, J., Schnell, N., Hengstenberg, W. 1999. The hprK gene of Enterococcus faecalis encodes a novel bifunctional enzyme: the HPr kinase/phosphatase. Mol Microbiol 31:59-66.

Mahr, K., Esteban, C.D., Hillen, W., Titgemeyer, F., Pérez-Martínez, G. 2002. Cross-communication between components of carbon catabolite repression of Lactobacillus case $i$ and Bacillus megaterium. J Mol Microbiol Biotechnol 4:489-494.

Marquez, J.A., Hasenbein, S., Koch, B., Fieulaine, S., Nessler, S., Russell, R.B., Hengstenberg, W., Scheffzek, K. 2002. Structure of the full-length $\mathrm{HPr}$ kinase/phosphatase from Staphylococcus xylosus at $1.95 \AA$ resolution: Mimicking the product/substrate of the phospho transfer reactions. Proc Natl Acad Sci USA 99:3458-3463.

Mazé, A., Boël, G., Poncet, S., Mijakovic, I., Le Breton, Y., Benachour, A., Monedero, V., Deutscher, J., Hartke, A. 2004. The Lactobacillus casei ptsHI47T mutation causes overexpression of a LevR-regulated but RpoN-independent operon encoding a mannose class phosphotransferase system. J Bacteriol 186: 4543-4555.

Mijakovic, I., Poncet, S., Galinier, A., Monedero, V., Fieulaine, S., Janin, J., Nessler, S., Marquez, J.A., Scheffzek, K., Hasenbein, S., Hengstenberg, W., Deutscher, J. 2002. Pyrophosphate-producing protein dephosphorylation by HPr kinase/phosphorylase: a relic of early life? Proc Natl Acad Sci USA 99: 13442-13447.

Monedero, V., Gosalbes, M.J., Pérez-Martínez, G. 1997. Catabolite repression in Lactobacillus casei ATCC 393 is mediated by CcpA. J Bacteriol 179:6657-6664.

Monedero, V., Kuipers, O.P., Jamet, E., Deutscher, J. 2001a. Regulatory functions of serine-46phosphorylated HPr in Lactococcus lactis. J Bacteriol 183:3391-3398.

Monedero, V., Poncet, S., Mijakovic, I., Fieulaine, S., Dossonnet, V., Martin-Verstraete, I., Nessler, S., Deutscher, J. 2001b. Mutations lowering the phosphatase activity of HPr kinase/phosphatase switch off carbon metabolism. EMBO J 20:3928-3937.

Phadtare, S. 2004. Recent developments in bacterial cold-shock response. Curr Issues Mol Biol 6:125-136.

Phadtare, S., Inouye, M., Severinov, K. 2002a. The nucleic acid melting activity of Escherichia coli $\mathrm{CspE}$ is critical for transcription antitermination and cold acclimation of cells. J Biol Chem 277:7239-7245.

Phadtare, S., Tyagi, S., Inouye, M., Severinov, K. 2002b. Three amino acids in Escherichia coli CspE surface-exposed aromatic patch are critical for nucleic acid melting activity leading to transcription antitermination and cold acclimation of cells. J Biol Chem 277: 46706-46711.
Porter, E.V., Chassy, B.M. 1988. Nucleotide sequence of the $\beta$-D-phosphogalactoside galactohydrolase gene of Lactobacillus casei: comparison to analogous $p b g$ genes of other Gram-positive organisms. Gene 62:263276.

Postma, P.W., Lengeler, J.W., Jacobson, G.R. 1993. Phosphoenolpyruvate:carbohydrate phosphotransferase systems of bacteria. Microbiol Rev 57:543-594.

Reizer, J., Novotny, M.J., Hengstenberg, W., Saier Jr, M.H. 1984a. Properties of ATP-dependent protein kinase from Streptococcus pyogenes that phosphorylates a seryl residue in $\mathrm{HPr}$, a phosphocarrier protein of the phosphotransferase system. J Bacteriol 160:333-340.

Reizer, J., Novotny, M.J., Stuiver, I., Saier Jr, M.H. 1984b. Regulation of glycerol uptake by the phosphoenolpyruvate-sugar phosphotransferase system in Bacillus subtilis. J Bacteriol 159:243-250.

Reizer, J., Panos, C. 1980. Regulation of $\beta$-galactoside phosphate accumulation in Streptococcus pyogenes by an expulsion mechanism. Proc Natl Acad Sci USA 77:5497-5501.

Reizer, J., Saier Jr, M.H. 1983. Involvement of lactose enzyme II of the phosphotransferase system in rapid expulsion of free galactosides from Streptococcus pyogenes. J Bacteriol 156: 236-242.

Rephaeli, A.W., Saier Jr, M.H. 1978. Kinetic analyses of the sugar phosphate:sugar transphosphorylation reaction catalyzed by the glucose enzyme II complex of the bacterial phosphotransferase system. J Biol Chem 253: 7595-7597.

Russell, R.B., Marquez, J.A., Hengstenberg, W., Scheffzek, K. 2002. Evolutionary relationship between the bacterial HPr kinase and the ubiquitous PEP-carboxykinase: expanding the P-loop nucleotidyl transferase superfamily. FEBS Lett 517:1-6.

Schumacher, M.A., Allen, G.S., Diel, M., Seidel, G., Hillen, W., Brennan, R.G. 2004. Structural basis for allosteric control of the transcription regulator CcpA by the phosphoprotein HPr-Ser46-P. Cell 118:731-741.

Senba, M., Kashige, N., Nakashima, Y., Miake, F., Watanabe, K. 2000. Cloning of the gene of $\beta$-N-acetylglucosaminidase from Lactobacillus casei ATCC 27092 and characterization of the enzyme expressed in Escherichia coli. Biol Pharm Bull 23:527-531.

-Tortosa, P., Declerck, N., Dutartre, H., Lindner, C., Deutscher, J., Le Coq, D. 2001. Sites of positive and negative regulation in the Bacillus subtilis antiterminators LicT and SacY. Mol Microbiol 41:1381-1393.

-Viana, R., Monedero, V., Dossonnet, V., Vadeboncoeur, C., Pérez-Martínez, G., Deutscher, J. 2000. Enzyme I and HPr from Lactobacillus casei: Their role in sugar transport, carbon catabolite repression and inducer exclusion. Mol Microbiol 36:570-584. 
Viana, R., Pérez-Martínez, G., Deutscher, J., Monedero, V. 2005. The glycolytic genes $p f k$ and $p y k$ from Lactobacillus case $i$ are induced by sugars transported by the phosphoenolpyruvate:sugar phosphotransferase system and repressed by CcpA. Arch Microbiol 183: 385-393.

Weber, M.H., Beckering, C.L., Marahiel, M.A. 2001. Complementation of cold shock proteins by translation initiation factor IF1 in vivo. J Bacteriol 183:7381-7386.

Weber, M.H.W., Marahiel, M.A. 2003. Bacterial cold shock responses. Sci Prog 86:9-75.

Wouters, J.A., Kamphuis, H.H., Hugenholtz, J., Kuipers, O.P., de Vos, W.M., Abee, T. 2000. Changes in glycolytic activity of Lactococcus lactis induced by low temperature. Appl Environ Microbiol 66:3686-3691.

Yamada, M., Saier Jr, M.H. 1988. Positive and negative regulators for glucitol $(g u t)$ operon expression in Escherichia coli. J Mol Biol 203: 569-583.
Ye, J.-J., Minarcyk, J., Saier Jr, M.H. 1996. Inducer expulsion and the occurrence of an $\mathrm{HPr}($ ser-P)-activated sugar phosphate phosphatase in Enterococcus faecalis and Streptococcus pyogenes. Microbiology 142:585592.

Ye, J.-J., Saier Jr, M.H. 1995a. Cooperative binding of lactose and the phosphorylated phosphocarrier $\mathrm{HPr}\left(\right.$ Ser-P) to the lactose $/ \mathrm{H}^{+}$ symport permease of Lactobacillus brevis. Proc Natl Acad Sci USA 92:417-421.

Ye, J.-J., Saier Jr, M.H. 1995b. Allosteric regulation of the glucose: $\mathrm{H}^{+}$symporter of Lactobacillus brevis: cooperative binding of glucose and $\operatorname{HPr}($ ser-P). J Bacteriol 177:1900-1902.

Ye, J.-J., Saier Jr, M.H. 1995c. Purification and characterization of a small membrane-associated sugar phosphate phosphatase that is allosterically activated by $\operatorname{HPr}(\operatorname{ser}(\mathrm{P}))$ of the phosphotransferase system in Lactococcus lactis. J Biol Chem 270:16740-16744.

Yebra, M.J., Monedero, V.,Zúñiga, M., Deutscher, J., Pérez-Martínez, G. 2006. Molecular analysis of the glucose-specific phosphoenolpyruvate:sugar phosphotransferase system from Lactobacillus case $i$ and its links with the control of sugar metabolism. Microbiology 152: 95-104.
Yebra, M.J., Pérez-Martínez, G. 2002. Cross-talk between the L-sorbose and D-sorbitol (D-glucitol) metabolic pathways in Lactobacillus casei. Microbiology 148:2351-2359.

Yebra, M.J., Veyrat, A., Santos, M.A., Pérez-Martínez, G. 2000. Genetics of L-sorbose transport and metabolism in Lactobacillus casei. J Bacteriol 182:155-163.

Yebra, M.J., Viana, R., Monedero, V., Deutscher, J., Pérez-Martínez, G. 2004. An esterase gene from Lactobacillus casei cotranscribed with genes encoding a phosphoenolpyruvate:sugar phosphotransferase system and regulated by a LevR-like activator and $\sigma^{54}$ factor. J Mol Microbiol Biotechnol 8:117-128.

Yoshida, K., Yamaguchi, M., Ikeda, H., Omae, K., Tsurusaki, K., Fujita, Y. 2004. The fifth gene of the iol operon of Bacillus subtilis, iolE, encodes 2-keto-myo-inositol dehydratase. Microbiology 150:571-580. 